\title{
BMJ Open Cluster randomised feasibility trial to improve the Control of Hypertension In Rural India (CHIRI): a study protocol
}

\author{
Michaela A Riddell, ${ }^{1}$ Rohina Joshi, ${ }^{2}$ Brian Oldenburg, ${ }^{3}$ Clara Chow, ${ }^{2,4}$ \\ K R Thankappan, ${ }^{5}$ Ajay Mahal, ${ }^{6,7}$ Nihal Thomas, ${ }^{8}$ Velandai K Srikanth, ${ }^{1}$ \\ Roger G Evans, ${ }^{9,10}$ Kartik Kalyanram, ${ }^{11}$ Kamakshi Kartik, ${ }^{11}$ Pallab K Maulik, ${ }^{12,13}$ \\ Simin Arabshahi, ${ }^{1}$ R P Varma, ${ }^{5}$ Rama K Guggilla, ${ }^{12}$ Oduru Suresh, ${ }^{2,11}$ G K Mini, ${ }^{5}$ \\ Fabrizio D'Esposito, ${ }^{3}$ Thirunavukkarasu Sathish, ${ }^{3}$ Mohammed Alim, ${ }^{12}$ \\ Amanda G Thrift ${ }^{1}$
}

To cite: Riddell MA, Joshi R, Oldenburg B, et al. Cluster randomised feasibility trial to improve the Control of Hypertension In Rural India (CHIRI): a study protocol. BMJ Open 2016;6:e012404. doi:10.1136/bmjopen-2016012404

- Prepublication history and additional material is available. To view please visit the journal (http://dx.doi.org/ 10.1136/bmjopen-2016012404).

Received 23 April 2016 Revised 2 August 2016 Accepted 16 August 2016

CrossMark

For numbered affiliations see end of article.

Correspondence to Professor Amanda Thrift; amanda.thrift@monash.edu

\section{ABSTRACT}

Introduction: Hypertension is emerging in rural populations of India. Barriers to diagnosis and treatment of hypertension may differ regionally according to economic development. Our main objectives are to estimate the prevalence, awareness, treatment and control of hypertension in 3 diverse regions of rural India; identify barriers to diagnosis and treatment in each setting and evaluate the feasibility of a community-based intervention to improve control of hypertension.

Methods and analysis: This study includes 4 main activities: (1) assessment of risk factors, quality of life, socioeconomic position and barriers to changes in lifestyle behaviours in 14 500 participants; (2) focus group discussions with individuals with hypertension and indepth interviews with healthcare providers, to identify barriers to control of hypertension; (3) use of a medicines-availability survey to determine the availability, affordability and accessibility of medicines and (4) trial of an intervention provided by Accredited Social Health Activists (ASHAs), comprising groupbased education and support for individuals with hypertension to self-manage blood pressure. Wards/ villages/hamlets of a larger Mandal are identified as the primary sampling unit (PSU). PSUs are then randomly selected for inclusion in the cross-sectional survey, with further randomisation to intervention or control. Changes in knowledge of hypertension and risk factors, and clinical and anthropometric measures, are assessed. Evaluation of the intervention by participants provides insight into perceptions of education and support of self-management delivered by the ASHAs.

Ethics and dissemination: Approval for the overall study was obtained from the Health Ministry's Screening Committee, Ministry of Health and Family Welfare (India), institutional review boards at each site and Monash University. In addition to publication in peer-reviewed articles, results will be shared with federal, state and local government health officers, local healthcare providers and communities.

Trial registration number: CTRI/2016/02/006678; Pre-results.

\section{Strengths and limitations of this study}

- The study is being conducted in three economically and developmentally diverse rural populations in Southern India, potentially enabling generalisability to many other regions within India.

- Data collection is standardised and consistent across the three settings.

- Validated tools allow for comparison with other studies conducted in India and similar settings in low to middle-income countries.

- Inclusion of a cross-sectional survey, a survey of availability of medicines and qualitative interviews with doctors and patients will allow triangulation of potential barriers to diagnosis, treatment and control of hypertension.

- The training programme delivered to nonphysician health workers has not been formally tested, thereby potentially limiting the efficacy of the intervention.

\section{INTRODUCTION}

Hypertension is the leading contributor to the global burden of disease and mortality. ${ }^{1}$ In 2000, 120 million Indians had high blood pressure (BP) and this figure is expected to increase to about 215 million by 2025. ${ }^{2}$ According to a recent systematic review of hypertension in India, the overall prevalence of hypertension was estimated to be nearly $30 \%$, with a significant difference in the overall pooled prevalence between urban $(33.8 \%$ (95\% CI $29.7 \%$ to $37.8 \%)$ ) and rural $(27.6 \%$ (95\% CI $23.2 \%$ to $32.0 \%)$ ) populations nationally. This disparity was particularly marked in West India (pooled prevalence urban $(35.8 \%$ (95\% CI $35.2 \%$ to $36.5 \%)$ ) and rural $(18.1 \%$ (95\% CI $16.9 \%$ to $19.2 \%)))^{3}$ With the rapid urbanisation of 
India, lifestyle changes, known to be associated with increased risk of hypertension, become more common. These lifestyle changes may be driving the convergence of the prevalence of hypertension between urban and rural India. ${ }^{4}$ Such convergence is observed in the pooled estimates, using random effect analysis, for South India where there were no detectable differences in the prevalence of hypertension between rural and urban populations (urban: $31.5 \%$ (95\% CI 23.6\% to $39.5 \%$ ) versus rural: $28.3 \%$ (95\% CI $21.4 \%$ to $35.1 \%$ ), $\mathrm{p}=0.62){ }^{3}$

Recent observations indicate that only approximately one-quarter of the people with a diagnosis of hypertension in rural Kerala receive treatment and, of this population, only $\sim 30 \%$ has their BP within the therapeutic target $^{5}{ }^{6}$ (systolic BP (SBP) $<140 \mathrm{~mm} \mathrm{Hg}$ and diastolic BP (DBP) $<90 \mathrm{~mm} \mathrm{Hg}$ ). ${ }^{7}$

There are many different barriers to the diagnosis and treatment of hypertension in urban and rural regions. These barriers likely comprise proximate determinants ${ }^{8}$ and operate at the individual and systems level. ${ }^{9}$ For example, awareness (diagnosis) of hypertension at the individual level may be influenced by distance to, and usage of, health services, physical inactivity and social factors. ${ }^{10}$ System level factors, which may influence awareness of hypertension, include the knowledge of risk factors by healthcare workers, availability of equipment for measuring BP and quality, availability and expertise of healthcare providers. Treatment of hypertension is influenced by age, distance to healthcare ${ }^{11}$ and socioeconomic position (SEP). ${ }^{12}{ }^{13}$ At the system level, treatment may be influenced by knowledge and implementation of current treatment initiation guidelines and availability of medicines. ${ }^{14}$ Control of hypertension is likely influenced individually by adherence to medication, SEP, health literacy and understanding of chronic disease and risk factors for chronic disease such as physical activity and tobacco use. ${ }^{11-13}{ }^{15}$ Within health systems, control of hypertension may be influenced by understanding of treatment guidelines, availability of medication and capacity to monitor and follow-up patients. ${ }^{14}$

There is also some emerging evidence that system and individual-level barriers may vary according to the stage of epidemiological, demographic and economic transition of different populations. ${ }^{8} 16$ For example, as the disease patterns in the population change rapidly, training of the health workforce may not be adequately up-to-date and basic diagnostic tools (such as BP machines) may also be lacking. In regions where the demographic and economic transition is more advanced, there is a greater prevalence of hypertension and thus a greater awareness of hypertension-related factors such as obesity and physical inactivity. ${ }^{17}$ In disadvantaged and poverty-stricken communities, where the population has not yet been exposed to economic development, the epidemiological transition is still at an earlier stage.
Disadvantaged communities still comprise the largest proportion of the population in resource-poor countries, but little is known about the awareness of hypertension or of the individual and system-level barriers to its diagnosis and treatment in these settings. An improved understanding of the awareness of hypertension in such disparate settings and the barriers to prevention, diagnosis and treatment will provide the critical knowledge base needed to overcome these barriers. The aims of this research are, within three diverse rural regions, to (1) estimate the prevalence, awareness, treatment and control of hypertension; (2) use these baseline data to develop strategies to better manage hypertension in rural communities in India; and (3) evaluate the feasibility of a community-based intervention to improve selfmanagement and control of hypertension.

The research protocol described herein comprises two phases in each of the three settings, with phase I having three parts:

Phase I: Baseline assessment

A. A baseline cross-sectional study to obtain information about the prevalence, awareness, treatment and control of hypertension.

B. Qualitative studies, comprising focus group discussions among individuals with hypertension and indepth interviews with healthcare providers, to identify individual and system-level barriers to control of hypertension.

C. A medicines-availability survey to determine the availability, affordability and accessibility of medicines used for treatment of hypertension, type 2 diabetes mellitus and secondary prevention of cardiovascular diseases.

Phase II: A feasibility study of an intervention to improve control of hypertension, developed based on the findings of the cross-sectional survey and qualitative studies. The intervention has two basic components: (1) peer group-based education and support for individuals with hypertension for self-management of BP; and (2) health services and workforce strengthening.

\section{Setting}

The study is being conducted in three diverse rural regions in Southern India, each of which is at a different stage of economic and demographic transition: Trivandrum in Kerala, the West Godavari District in Northern Andhra Pradesh (AP) and the Rishi Valley region in Southern AP (see online supplementary figure S1).

Trivandrum, Kerala (late transition): Kerala is the most advanced state in demographic and epidemiological transition in India. ${ }^{18}$ Life expectancy is 76.4 years ${ }^{19}$ and literacy is $93.9 \% .^{20}$ Approximately half of the population in the state resides in non-urban areas. ${ }^{21}$ Changes in cultivation patterns from food crops to more profitable cash crops and large-scale international migration has rendered Kerala a wealthier and a less agrarian state than the rest of India. ${ }^{19} 22$ 
West Godavari, AP (medium transition): The Western Godavari study region comprises 897 villages. Life expectancy in Andhra Pradesh in 2001-2006 was 62.8 years for men and 65 years for women. ${ }^{23}$ In $2011 \sim 75 \%$ of the population in West Godavari was literate, and the majority of the residents $(79.5 \%)$ lived in rural areas. ${ }^{24}$

Rishi Valley, AP (early transition): This rural site is located in the Kurabalakota Mandal, which contains six villages, in the Chittoor District near the South Western border of AP. Approximately 38000 residents in Kurabalakota Mandal reside in 221 hamlets (habitations/subvillages). Hamlets are the smallest administrative geographic units in this region. The population of this Mandal are largely subsistence farmers and are economically disadvantaged with an average monthly household income well below the global standard for poverty. Approximately half the population in this region is estimated to have no formal schooling. ${ }^{25}$

\section{HYPOTHESES}

We hypothesise that:

1. Knowledge/awareness of the presence of hypertension and about risk factors associated with hypertension is greater in the late transition region than in the early transition region.

2. Prior BP measurement is less common in the early transition region (Rishi Valley) than in the late (Trivandrum) and medium transition region (West Godavari).

3. In those previously identified as having hypertension, costs of treatment are the greatest barrier to ongoing management of hypertension in all settings.

4. Poor management of hypertension is more common in women, people living below the poverty line and in those who did not finish high school.

5. High salt intake is a major risk factor for hypertension in men and women in the late transition region, but its effect is limited to men in the early transition setting.

6. A community-based peer group education and selfmanagement programme conducted by Accredited Social Health Activists (ASHAs) is feasible.

\section{METHODS AND ANALYSIS}

\section{Study design/sampling frame}

Wards/villages/hamlets of a larger mandal (also known as taluk) were identified as the primary sampling unit (PSU). At each study site, these PSUs were then randomly selected for inclusion in the cross-sectional survey (Phase I of the study, figure 1) using computergenerated random numbers. For Trivandrum, the PSU is wards, for West Godavari, the PSU is villages and for the Rishi Valley site, the PSU is hamlets (habitations) (see online supplementary figure S2A-C for site-specific sampling frames).

\section{la: Baseline cross-sectional survey}

A cross-sectional survey was initially conducted to quantify the burden and awareness of hypertension and examine how the barriers to diagnosis and management differ between settings. Recruitment for this study started in January 2014 and was completed in December 2015. Approximately 14500 adults living in the study areas have been selected to provide data for the baseline survey. The role of gender, socioeconomic deprivation and education on the diagnosis and management of hypertension in each of the three rural areas is being explored. The data have been used to design the intervention component of the study.

\section{Recruitment to cross-sectional survey}

Population censuses (specifically completed for this study or existing polling booth registers) at each site were used to randomly select potential participants. In
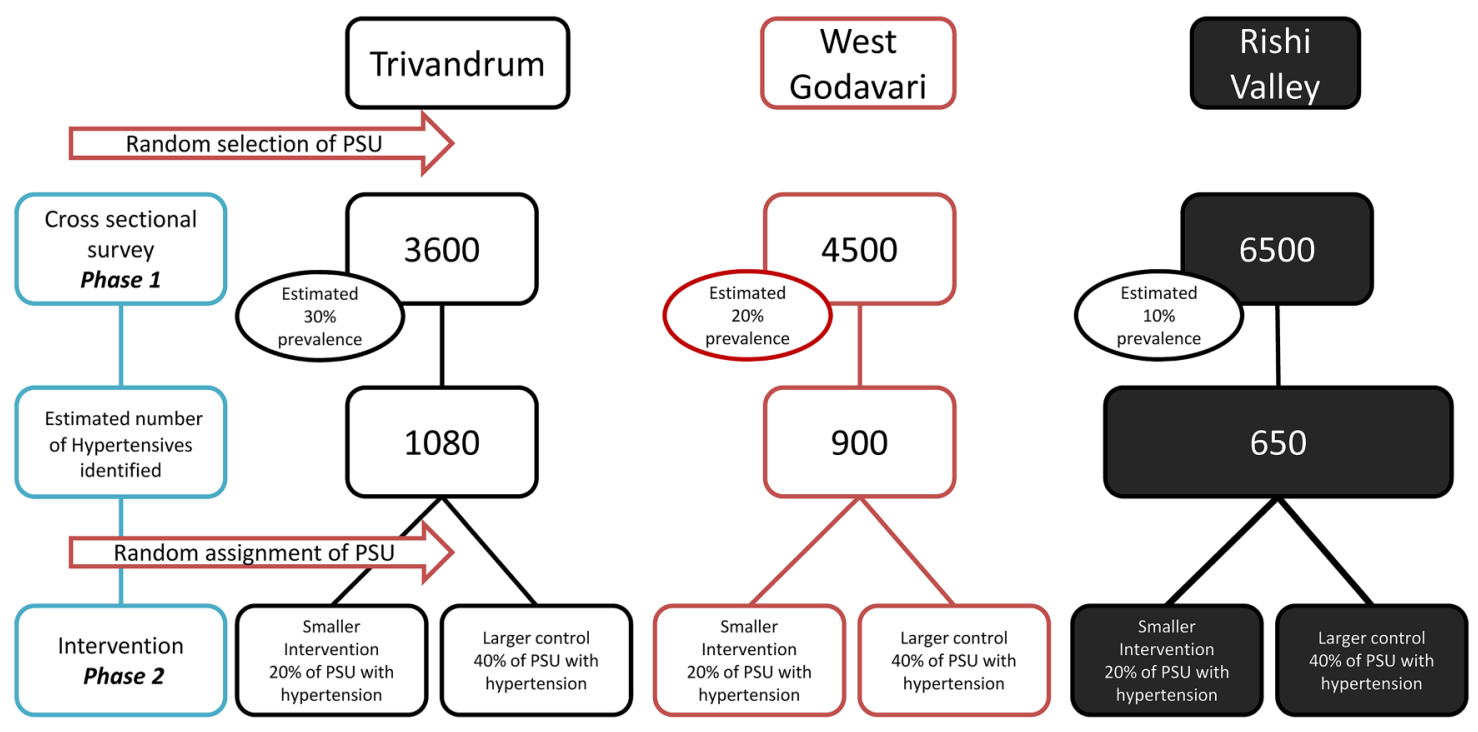

Figure 1 CHIRI study design outlines the approach taken to select and survey the populations. PSU, primary sampling unit. 
Trivandrum and West Godavari, sampling was stratified by age and sex using this approach. However, due to structural factors related to distance between hamlets and demography, this was not feasible in the Rishi Valley region. Residents of the PSU aged at least 18 years were eligible for recruitment to the cross-sectional survey.

Trivandrum: Among the 14 districts in Kerala, Trivandrum district was selected based on its proximity to the collaborating institute (Sree Chitra Tirunal Institute for Medical Sciences and Technology). Chirayinkizhu taluk was selected randomly from the four taluks in Trivandrum district. Of the 22 Panchayats (local administrative body in rural areas) within Chirayinkizhu taluk, 10 were randomly selected. From each of the selected Panchayats, one ward (the smallest geographic unit of a Panchayat) was randomly selected. In each ward, using the polling booth list, the total number of individuals was divided into 12 age and sex groups (18-24, 25-34, 35-44, 45-54, 55-64 and 65+). From each group, 30 individuals were randomly selected to get a total sample of 360 in each ward. Thus, 3600 participants were selected in the Trivandrum district to participate in the cross-sectional survey. Additional sampling was conducted (10 from each age and sex band) to replace those participants who had migrated, died or refused to participate.

West Godavari: A sampling frame was developed by mapping all 17 Primary Health Centres (PHCs) within a $50 \mathrm{~km}$ radius around the town of Bhimavaram. We excluded those PHCs participating in other studies conducted by the George Institute and randomly selected 10 of the remaining PHCs. All the villages serviced by each PHC were included in the list. Villages with fewer than 3000 residents were excluded. One village was then randomly selected from each PHC, resulting in inclusion of 10 villages. Mapping all the selected villages enabled generation of an age and sex population list with house addresses. Population lists from the mapping were used to randomly select 4500 individuals over the age of 18 years from each participating village. Additional sampling was conducted to replace those participants who had migrated, died or refused to participate. Sampling was stratified by 12 groups defined by age (18-24, 25-34, $35-44,45-54,55-64,65+)$ and sex with the goal of including comparable numbers of individuals from each group.

Rishi Valley region: The study population comprised the six villages of the Kurabalakota Mandal in the Chittoor District, Southern AP. These villages comprised 221 hamlets or small habitations.

Hamlets were stratified by population size (small, medium and large) and then 139 were randomly selected in accordance with the sampling strategy using computer-generated random numbers (generated at Monash University). This was to ensure sampling of approximately equal numbers of hamlets from each size stratification. Six hamlets were excluded due to migration of population. We also excluded the hamlet in which the Rishi Valley Rural Education Centre was located because the population was largely transient, comprising teachers and students who reside in the hamlet only during school time. A study centre was set up in a communal area of the hamlet convenient for all the residents. All residents aged at least 18 years were invited to participate in the cross-sectional survey. Research officers ensured that all residents were informed of the presence of the study team in the habitation by house-to-house notification and encouragement to attend.

\section{Data collection/measurement}

The instruments and measurements chosen for this project are based on recommendations from the WHO STEP-wise approach to disease surveillance (WHO STEPS ${ }^{26}$ and other validated tools for quality of life, SEP and barriers to changes in alcohol and tobacco behaviour as listed in online supplementary table S1. The list of measures comprise (1) basic demographic information, including age, income, gender, marital status, religion, number of children and type of work undertaken; (2) lifestyle-related factors such as physical activity, tobacco use and alcohol consumption, dietary factors, including cooking practices and use of salt, stress and overcrowding; (3) knowledge about hypertension and its risk factors, awareness of hypertensive status and reports of the timing and outcome of prior BP measurements; and (4) further details about the use of medications (allopathic and AYUSH or other traditional therapies), barriers to treatment, including access, cost, adoption of lifestyle factors and compliance with medication use (see online supplementary table S1 for full list of variables). Questionnaires were developed in English and then translated into the site-specific language (Telugu (AP), Malayalam (Kerala)) and back translated to detect and correct errors.

Standardised clinical measurements are collected as follows; arterial BP and heart rate are measured after the participant has sat quietly for at least $15 \mathrm{~min}$. BP is measured at least three times at 3 min intervals using the appropriate cuff size and a Digital Automatic Blood Pressure Monitor (OMRON HEM-907, OMRON Healthcare Company, Kyoto, Japan) according to the WHO STEPS protocol, modified only by using the right arm for all measurements. ${ }^{26}$ Measurement continues until two consecutive readings differ by $<10 \mathrm{~mm} \mathrm{Hg}$ systolic and $<6 \mathrm{~mm} \mathrm{Hg}$ diastolic, with a maximum of five measurements. The mean of the last two consecutive measurements are used to define hypertensive status. Height is measured to the nearest $0.1 \mathrm{~cm}$ using a portable stadiometer (213, Seca, Hamburg, Germany). Weight is measured to the nearest $0.1 \mathrm{~kg}$ using a portable digital weighing scale (9000SV3R, Salter, Kent, UK). Waist and hip circumference is measured using a springloaded tension tape (Gulick M-22C, Patterson Medical, Illinois, USA) in a private setting. In accordance with the WHO STEPS protocol, ${ }^{26}$ waist circumference is 
measured at the midpoint between the lowest rib and upper point of the iliac crest and at the end of normal expiration and hip circumference is measured at the maximum protrusion of the buttocks.

To ensure standardisation, data collectors are trained in collection of anthropometric and BP measurements in accordance with the WHO STEPS protocol. ${ }^{26}$ This training, conducted by the project manager for at least 5 days, is to ensure consistency of data collection between sites. Training is provided in a similar manner to ensure that questionnaire administration is also consistent across all sites. A study-specific training manual containing step-by-step procedures for all data collection (anthropometric and survey administration) is provided to each data collector. Data collection at each site is further monitored by site supervisors. Follow-up training by the project manager and/or site supervisor is also undertaken at each site $\sim 1$ month after initiation of data collection to ensure that data collection methods are implemented according to the protocol.

Definitions: Participants whose measured mean SBP is $\geq 140 \mathrm{~mm} \mathrm{Hg}$ and/or mean DBP is $\geq 90 \mathrm{~mm} \mathrm{Hg}$ or who are taking medication for lowering BP are defined as hypertensive. ${ }^{7}$ Waist circumference is deemed high when $>80 \mathrm{~cm}$ in women and $>90 \mathrm{~cm}$ in men. A body mass index (BMI) $\geq 25$ to $29.99 \mathrm{~kg} / \mathrm{m}^{2}$ is defined as overweight and BMI $\geq 30 \mathrm{~kg} / \mathrm{m}^{2}$ as obese according to the revised BMI classification. ${ }^{27}$

\section{Outcomes}

Sociodemographic and economic characteristics of each population are determined, including gender, SEP, education, income and expenditure. Information on usage of healthcare, physical activity, tobacco use and consumption of alcohol are also collected. Primary outcomes for Phase Ia of the study are prevalence, awareness, treatment, control, knowledge of hypertension and associated risk factors. We compare knowledge of hypertension, previous measurement of BP and barriers to treatment and management (such as cost, education and SEP) of hypertension across study sites. Good (BP $<140 / 90 \mathrm{~mm} \mathrm{Hg}$ ) or poor ( $\mathrm{SBP} \geq 140 \mathrm{~mm} \mathrm{Hg}$ or DBP $\geq 90 \mathrm{~mm} \mathrm{Hg}$ ) control of hypertension is assessed in relation to lifestyle factors (physical activity, use of tobacco and consumption of alcohol), dietary factors (including salt intake) and healthcare usage. Attitudes to healthy behaviour change are also assessed.

\section{Qualitative studies to identify barriers (phase $\mathrm{Ib}$ )}

To further investigate barriers to diagnosis, treatment and control of hypertension, focus group discussions with people having hypertension are used to explore peoples' experiences with healthcare systems and their perceptions and beliefs about hypertension in accordance with predetermined and shared exploratory questions (see online supplementary table S2 for the interview guide). Up to 4 focus groups involving up to 10 people with hypertension in each focus group, identified in the cross-sectional survey as being aware of their hypertension status, are conducted at each site (refer online supplementary figure S2A-C). Focus groups are voice recorded and a second research officer takes notes throughout the session. The recordings are translated and transcribed into English and checked for accuracy with the research notes. Data analysis is carried out using the process described by Green et al. ${ }^{28}$ We use a socioecological approach to the identification of themes, which considers the complex interplay between individual, social and systemic factors. ${ }^{29-31}$

Indepth interviews are carried out with healthcare providers (doctors, staff nurses, ANMs/ASHAs) at each site to explore management of hypertension, and perceptions of the healthcare system in relation to screening and management of hypertension and other chronic conditions (see online supplementary tables S3 and S4 for the interview guides).

\section{Medicine pricing and availability: (phase Ic)}

To investigate the availability and price of medicines for hypertension and other related non-communicable diseases (NCDs) (eg, cardiovascular diseases (CVD), type 2 diabetes), a cross-sectional survey of price and availability of essential medicines is undertaken. Included in this survey are public (hospitals, clinics and health facilities), private (licensed retail pharmacies and licensed drug stores) and 'other' sector medicine outlets (facilities selling medicines at subsidised prices to all patients) located in the sampling frames of the CHIRI study sites in AP and Kerala (refer online supplementary figure S2A-C). The medicines selected for review are those used for treatment of hypertension, secondary prevention of CVD, and treatment of type 2 diabetes mellitus and that are listed in the WHO Model List of Essential Medicines, ${ }^{32}$ National List of Essential Medicines of India, ${ }^{33}$ Essential Medicines List of the Government of (unified) Andhra Pradesh and the Rational Drug List 2012-2013 of Kerala Medical Services Corporation. ${ }^{34}$ The study methods have been developed based on the WHO/HAI (Health Action International) methodology for measuring medicine prices, availability, affordability and price components. ${ }^{35}$ The survey is being conducted across 20 public, 16 private and 2 'other' sector pharmacies in the three regions.

Phase II: feasibility of a community-based programme for management of hypertension facilitated by ASHA

Phase II of the study is a feasibility trial which incorporates random allocation of $\sim 20 \%$ of the PSUs to the intervention and $40 \%$ of the PSUs to the control condition (figure 1). Specifically for the Trivandrum region, 2 of 10 wards are allocated to the intervention and 4 of 10 wards are allocated to the control condition. For the West Godavari region, 2 of 10 villages are allocated to the intervention and 4 of 10 villages are allocated to the control condition. For the Rishi Valley region, hamlets in one of six villages are allocated to the intervention 
and hamlets in two of six villages to the control condition.

Allocation of only a subset of the initial PSUs was necessary due to budgetary and programme timeline constraints. Random allocation of the PSUs to intervention or non-intervention conditions is undertaken by the principal investigator (figure 1). As this is a feasibility study, the sample size for those receiving the intervention is not powered to determine effectiveness. However, changes in individual measurements over time are assessed. The intervention addresses those factors that are identified during the qualitative and cross-sectional studies which contribute to control of hypertension in these settings and includes management and control strategies aimed at the individual, health service delivery and policy levels.

\section{Blinding}

As this is a behavioural intervention programme, the people delivering the intervention cannot be blinded to the intervention group. However, participants in the control regions remain unaware of the intervention programme, and outcome assessors are blinded to the intervention allocation of participants.

\section{Recruitment and eligibility}

Community members from the randomly selected PSUs, who are identified as hypertensive in the cross-sectional study, are invited to participate in the intervention and control arms in accordance with eligibility criteria given below (also see figure 2). Recruitment started in January 2016, and the intervention is expected to be completed in June 2016. Participants are approached at their homes after identification, and recruited and consented during this visit. For the intervention sites, the list of consented participants is provided to the ASHAs from those villages. Eligible participants are those who:

1. Indicate they are aware of being hypertensive in the cross-sectional survey.

2. Are identified as having an average SBP of $\geq 140 \mathrm{~mm} \mathrm{Hg}$ and/or DBP $\geq 90 \mathrm{~mm} \mathrm{Hg}$ at the crosssectional survey, subsequently attend their primary healthcare provider and are then formally clinically diagnosed with hypertension. Verification occurs by sourcing the medical record, contacting the healthcare provider or by confirmation of use of medication(s) for hypertension (as observed).

3. Have an average BP of $\geq 140 \mathrm{~mm} \mathrm{Hg} \mathrm{SBP}$ and/or $\geq 90 \mathrm{~mm} \mathrm{Hg}$ DBP during the cross-sectional survey and then, at the time of recruitment to the intervention (or control), have their BP remeasured, and are found to still have an average $\mathrm{BP}$ of $\geq 140 \mathrm{~mm} \mathrm{Hg}$ SBP and/or $\geq 90 \mathrm{~mm} \mathrm{Hg} \mathrm{DBP.}$

4. Are taking medication(s) for hypertension (including diuretics, ACE inhibitors, angiotensin II antagonists, $\beta$ blockers, $\mathrm{Ca}^{2+}$ channel blockers or renin inhibitors.)

\section{Intervention components}

The intervention arm incorporates a community-based self-management and education support group, led by

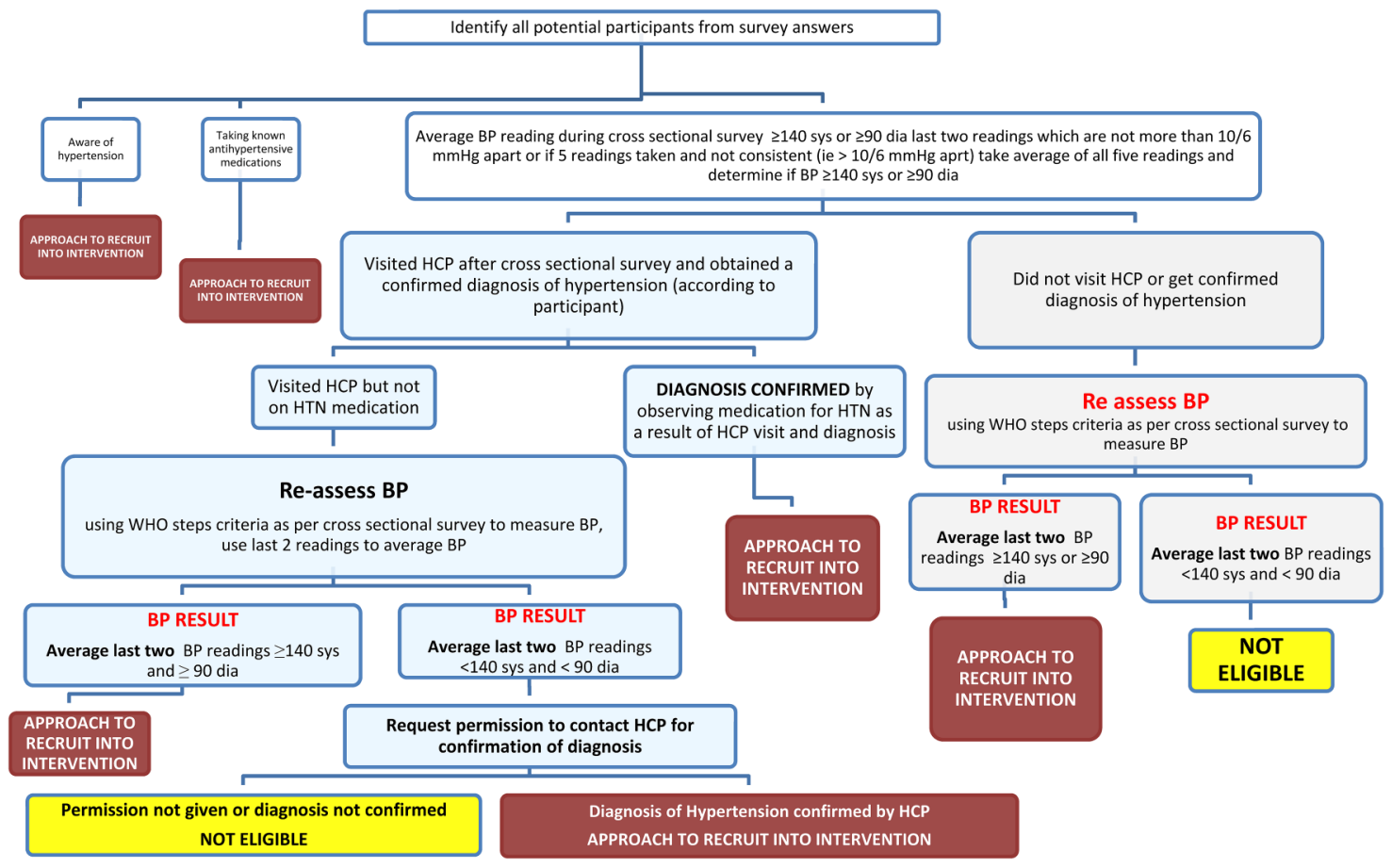

Figure 2 Recruitment flow chart for intervention study. Residents of the primary sampling unit who participated in the baseline cross-sectional survey are eligible to be recruited into the feasibility trial (intervention and control arms) based on the criteria and recruitment flow chart depicted here. BP, blood pressure; DIA, diastolic; HCP, healthcare provider; HTN, hypertension; SYS, systolic. 
ASHAs, every 2 weeks for 3 months (ie, six meetings). ASHAs are female lay health workers, residing in each village, most of whom have completed secondary schooling. Their work accountability primarily lies within the purview of the Village Health Sanitation and Nutrition Committee (VHSNC) which is a committee formed at the revenue village level and acts as a subcommittee of Gram Panchayat. They are compensated for their time in specific situations (attending training and meetings) and given incentives under various national health programmes, predominantly for maternal and child health.

Content for the intervention components was driven by preliminary analysis of the cross-sectional data which reflected poor knowledge of hypertension. Furthermore, principles of the Chronic Disease Self-Management Program (CDSMP) as described by Lorig et $a l,{ }^{36}$ and found to significantly contribute to improved selfmanagement, were incorporated into the programme content. ${ }^{37}$ Content includes strategies to increase knowledge and understanding of the disease, promote healthy behaviour change and clinical interaction through goal setting. At each group meeting, participants are weighed, have their BP measured and receive self-management and lifestyle education (figure 3) from locally sourced 'expert' advisers. These advisers may include, but are not limited to, clinicians from the PHC servicing the region, pharmacists and nutritional advisors.

\section{ASHA training}

For the intervention, ASHAs in each location are trained to deliver the self-management sessions of the intervention and to collect data regarding the implementation of the intervention. Training of ASHAs is standardised and undertaken by the site supervisors at each site in accordance with the study-specific ASHA training manual to ensure consistency of training between sites. Training of ASHAs was first piloted at the Rishi Valley site using four volunteer ASHAs not involved in the implementation of the intervention. Pilot training was conducted by the Project Manager and included each site supervisor using 'train the trainer' principles. ${ }^{38}$ Materials and resources for training ASHAs, as well as standardised resources and education material for delivering the intervention, were initially developed in English. ASHAs involved in the pilot training provided important feedback that enabled us to refine the educational resources for ASHAs and participants. Once these resources were finalised, they were translated/back translated into site-specific language (Telugu and Malayalam).

At the beginning of the first training session, the knowledge of ASHAs regarding hypertension and other NCDs and their skills in measuring BP and recording weight are assessed. The 5-day training regimen includes education about NCDs that is based on, and complementary to, the existing ASHA training Module for

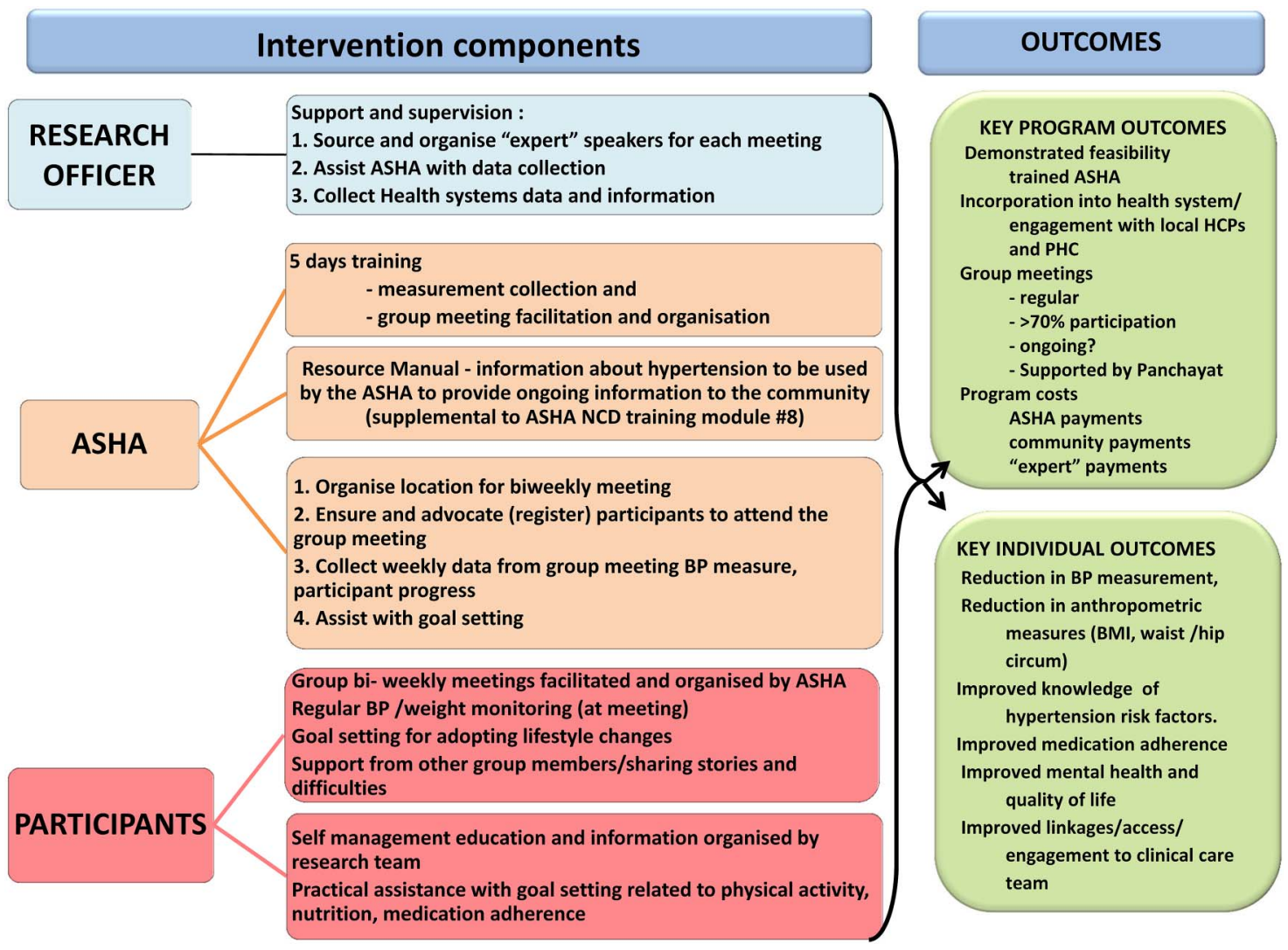

Figure 3 Feasibility trial intervention components and proposed outcomes. ASHA, Accredited Social Health Activist; BMI, body mass index; HCP, healthcare provider; PHC, primary health centre. 
NCDs (Module \# 8), ${ }^{39}$ measurement and recording of $\mathrm{BP}$ and weight, and maintaining records of each meeting (attendance, measurements, meeting content, record problems or issues faced by participants). Additionally, ASHAs are trained to deliver the educational material regarding hypertension and self-management using pictorial flip-chart resources developed for the intervention. ASHAs are also trained to initiate and support self-management of hypertension by the participants through goal-setting. Incentives for ASHAs are based on remuneration under the schedule for Village Health Sanitation and Nutrition Committee Activities ${ }^{40}$ as well as Village Health and Nutrition Days. ${ }^{41}$ In the two regions in Andhra Pradesh, the ASHAs are incentivised to promote attendance by as many participant and support members as possible, via payment of 200 Indian rupees per meeting if more than $75 \%$ of enrolled participants attend. Thus, those that do not attend one meeting will be followed up by an ASHA and encouraged to attend the next meeting. In the Kerala site, the ASHAs are paid according to remuneration under the schedule for Village Health Sanitation and Nutrition Committee Activities ${ }^{40}$ as well as Village Health and Nutrition Days, ${ }^{41}$ and were not paid extra when more than $75 \%$ of participants attended.

\section{Self-management education and group-based support}

Each community group comprises up to 20 individuals with hypertension. The community-based support group is supported and promoted by the Panchayat at each site. The Sarpanch (the elected head of village) provides a letter of support and encouragement for the intervention activities. This letter is enclosed with the letter of invitation to participate in the intervention. The facilitator of the group, either the appointed village ASHA (Trivandrum, West Godavari and Rishi Valley regions) or a person with equivalent qualifications employed for the project (West Godavari region), is assisted by a member of the research team at each meeting. The facilitator of the group organises and schedules meetings, collects data during the meeting and submits these data to the research team.

Each of the six meetings lasts $\sim 90 \mathrm{~min}$, and consists of various topics related to hypertension (table 1). Next of kin or additional support persons are encouraged to accompany and support the person with hypertension at each group meeting.

Because of the nature of the intervention, a community-based peer support group with self-management education, no data safety monitoring committee is required.

The community-based groups are used to educate group members about hypertension as well as implement and enhance strategies for self-management of hypertension and related NCDs (table 1 and figure 3). Resources developed specifically for the intervention are primarily pictorial to ensure consistency of information at each site and to account for disparities in educational levels across the three sites. ${ }^{25}$

\section{Health systems activities/interaction}

This feasibility study incorporates interaction and active involvement with providers from the structured health systems. Involvement of the health providers (at primary, community and subhealth centre levels) may strengthen and complement the group-based intervention as well as enhance follow-up interactions with the health system (including clinical and pharmaceutical services). The facilities of healthcare providers serving the communities, selected for the baseline study, are assessed to identify how equipment and staffing could be supported to improve diagnosis, treatment and ongoing management of hypertension.

\section{Analysis of the costs of the intervention}

Costs of the intervention will be assessed in terms of programme costs such as ASHA incentives and meeting

\begin{tabular}{|c|c|c|}
\hline $\begin{array}{l}\text { Meeting } \\
\text { number }\end{array}$ & Topic & Detailed information provided \\
\hline 1 & Introduction & $\begin{array}{l}\text { Education session: what is hypertension?, risk factors, chronic nature of disease, } \\
\text { 'know your numbers', etc to be carried out by a local healthcare provider }\end{array}$ \\
\hline 2 & $\begin{array}{l}\text { Self-management } \\
\text { education }\end{array}$ & $\begin{array}{l}\text { Risk factors and modifiable activities to improve control/management. Importance } \\
\text { of medication adherence }\end{array}$ \\
\hline 3 & Physical activity & Incorporating physical activity into your day, including group physical activity \\
\hline 4 & Nutrition and diet & $\begin{array}{l}\text { Importance of salt reduction (including recipes), alcohol reduction, dietary } \\
\text { assistance, increased fruit and vegetable consumption (it is especially important } \\
\text { for women to be given strategies to save some vegetables and meat for } \\
\text { themselves) }\end{array}$ \\
\hline 5 & $\begin{array}{l}\text { Practical } \\
\text { self-management }\end{array}$ & $\begin{array}{l}\text { Practical ways to improve your control/management (medication diary/reminder } \\
\text { system, etc). A pharmacist may attend and provide information about drug } \\
\text { availability }\end{array}$ \\
\hline 6 & $\begin{array}{l}\text { Next steps/continuation } \\
\text { plans }\end{array}$ & Review, changes made, ongoing difficulties, ongoing group activities \\
\hline
\end{tabular}


set-up costs. Cost of developing resources including development time and production of resources will inform this analysis.

\section{Control group}

At the time of the initial data collection, all participants with elevated BP (SBP $\geq 140 \mathrm{~mm} \mathrm{Hg}$ and/or DBP $\geq 90 \mathrm{~mm} \mathrm{Hg}$ ) in the control sampling units are informed that their BP is elevated and are advised to visit their local health provider for further investigation. Additionally, these participants are provided basic nutritional advice such as reducing dietary salt, reducing use of palm oil, increasing intake of fruits and vegetables, increasing physical activity and reducing use of tobacco and alcohol. Participants who were previously aware of their hypertensive status are advised to revisit their doctor for review of their medication and to take their medication as told by their doctor. No further care or advice is provided during the intervention period.

At the end of the intervention period, the participants with hypertension in the control sampling units are revisited for outcome measures, as outlined below. At this time, these participants receive further advice and recommendations regarding their hypertension status. Furthermore, they are provided with pictorially based educational material about hypertension and how to manage the disease.

\section{Outcomes of the intervention}

Participants attending the group meetings are revisited $\sim 6-8$ weeks after the last meeting to complete final data collection. In these participants, and in those in the control arm, we remeasure BP and anthropometry and reassess healthcare usage, attitudes to behaviour change and activities related to self-management of hypertension (see online supplementary table S1). Changes in continuous and categorical variables are assessed relative to their values determined at the time of the crosssectional survey. These include SBP, DBP, waist and hip circumference and weight. Medication initiation and any dosage changes (observed and documented) are recorded. Medication adherence to BP-lowering medication is assessed in accordance with the Hill-Bone Compliance Adherence Survey. ${ }^{42}$ This survey is used to assess the use of prescribed medication over the prior 2-week period. ${ }^{42}$ Knowledge about hypertension and associated risk factors relating to hypertension is reassessed, along with physical activity over the 2 weeks prior to final administration of the questionnaire. Barriers to attending the meetings are assessed, as are engagement and usage of health services during the period of the intervention.

Perceptions by participants of the level of support obtained from the ASHA ${ }^{43} 44$ are assessed in the final survey using a three scale response (not at all/some of the time/all of the time). Usefulness of advice and selfmanagement assistance received by the participant is assessed using a seven-point Likert scale (a little useful to extremely useful). Encouragement and support received, as perceived by the participant, resulting from various group meeting activities is assessed using a fourpoint response (no support, a little, moderate, a lot of support).

After each meeting, ASHAs complete a meeting report to aid the assessment of fidelity of the meeting structure and content to the protocol (see online supplementary table S5). This report includes details of the meetings such as the number of enrolled participants and community members attending the meeting, and major activities undertaken during the meeting. Additional activities by the ASHA to extend the healthy behaviour messages, such as tobacco cessation and physical activity, as well as behaviours specific to the management of hypertension from the meeting to the wider community by the ASHAs is also measured categorically (Yes/No). This measure captures support/interactions of ASHAs with meeting participants outside the meeting and their discussions of the meeting content with other members of the community and/or with community leaders or health service members.

Members of the research team also complete a report after each meeting to provide further information about the meeting activities and detail the shared experiences or difficulties of the participants in managing their hypertension or achieving their goals (see online supplementary table S5). These reports are used to assess the fidelity of the implementation of the intervention to the protocol.

System outcomes of the programme are obtained via surveys of ASHAs at the end of the intervention (and compared with surveys conducted at the initiation of the intervention) to assess changes in knowledge of ASHAs (see online supplementary table S5). We also assess the integration or engagement of health providers in the programme by assessing their participation during the intervention. Furthermore, participants provide information about other providers of assistance and information/advice (healthcare team and family/friends/ community) and assess meeting activities (goal setting, attendance, problem solving) for delivering selfmanagement support. The overall response rate (people with hypertension in the cross-sectional survey, number consented to participate in meetings) is calculated and participants who discontinue the programme are asked to complete a programme evaluation form and to undertake final measurements at the end of the intervention.

\section{Data management}

All assessments and data forms are checked on the day of completion. Any forms with missing data or inconsistencies are returned to the Health Worker for completion. Data are coded and entered as the study progresses. Edit checks are performed and data verified as necessary. 
All forms are designed in TeleForm Elite V.10.5. Completed questionnaires are electronically scanned into a computer using a Tagged Image File Format (TIFF). These data are then transferred to Monash University and uploaded into a Microsoft Access database using TeleForm. The database contains no identifying information and is housed on a secure server at Monash University. All principal investigators will have access to the deidentified final data.

\section{Data analysis}

Analysis will be based on intention to treat. For individual outcomes, proportions will be compared using $\chi^{2}$ test and continuous measures will be compared using Student's unpaired t-test. Univariable analysis will also be undertaken using logistic and linear regression. Multivariable analyses will be adjusted for age, sex and study site. Considering the unequal distribution of age and sex in the participating population, appropriate sampling weights will be applied to all data analyses.

Knowledge/awareness of the presence of hypertension and about risk factors will be calculated using the known (and measured) prevalence of hypertension in each population. Between-group differences in knowledge, previous measurement of hypertension and barriers to treatment and their change over time will be compared using $\chi^{2}$. Logistic regression (appropriate for the binary outcome variable of the presence/absence of $\mathrm{BP} \geq 140 / 90 \mathrm{~mm} \mathrm{Hg}$ ) will be used to study the factors associated with control of hypertension. Those factors identified as either potentially significant $(p<0.20)$ or biologically relevant will then be introduced into multivariable regression analyses in a backward stepwise fashion to construct a predictive model for good management of hypertension. This technique enables the assessment of categorical variables (eg, gender) as well as continuous variables (eg, age). The scales of the continuous covariates will be checked for suitability using fractional polynomial plots. Collinearity between variables will be evaluated using partial correlations. In addition, routine diagnostic tests (Hosmer-Lemeshow test) will be used to validate the fitted model. We will test efficacy of the intervention by analysis of covariance. This allows us to adjust for baseline differences between groups.

\section{Trial registration}

We applied for registration of the feasibility trial with the Clinical Trials Registry of India (CTRI) on 28 September 2015, 2 months before the first patient was enrolled. Our registration number (CTRI/2016/02/006678) was obtained from the CTRI on 25 February 2016, without any changes to the study design outlined in our original application. Such delays are very common in India and so it is usual practice to start recruiting patients before the final registration number is received.

\section{Dissemination}

All participants are provided a written participant information sheet and informed consent form in the local language. The study research staff at each site is responsible for obtaining informed consent. For those participants who are illiterate, the participant information sheet is read out to them. All participants sign (thumb print for those who are illiterate) the informed consent form.

Each consenting participant is assigned a study identification number. To maintain confidentiality all questionnaires, and other study documents, include only this identification number, with no identifying information present.

Feedback from study phases $1 \mathrm{~b}$ and $1 \mathrm{c}$ will be consolidated after the intervention and disseminated to public health systems to inform health provision services. Based on medicines availability (phase 1c) and qualitative feedback from focus groups and in depth interviews (phase 1b) a treatment algorithm/guideline which is consistent with the Indian Hypertension Management Guidelines ${ }^{7}$ will be developed. In order to strengthen the integration of community-based and delivered education programmes for self-management of health into the local primary healthcare system, we will disseminate the following information to benefit local health services through:

1. sharing of information gained from the crosssectional survey;

2. developing resources for use by health system staff for assessing and treating hypertension;

3. providing details to health centres about the resources and training they require to support such an intervention.

We will also disseminate the following information to improve availability of pharmaceutical preparations:

1. informing pharmacies about medicine availability and possibilities for providing medications to suit communities enrolled in the intervention;

2. discussing options for enhancing or improving medication adherence (by addressing availability, dosing and packaging options).

Results will be shared with the Ministry of Health \& Welfare and officials of the National Health Mission and relevant local healthcare providers and communities at each of the sites. Further dissemination of the results to research, clinical and health communities will be pursued via international peer-reviewed journal articles and conference presentations. The Global Alliance for Chronic Diseases (GACD) will also be informed of the findings of the study.

\section{DISCUSSION}

Comprehensive assessment of the barriers to control of hypertension across three diverse settings will provide important information about the diversity of barriers to care across these settings and how these might most 
appropriately be addressed in the future. Development of an intervention programme, based on the individual and system barriers identified and tailored to the specific needs of each area included in the study, may provide a solution to address the current deficiencies in managing hypertension across rural regions. We will also be able to determine the feasibility of using ASHAs to deliver a community group-based self-management programme for management of hypertension. Task shifting disease management and control from physicians to non-physician health workers is increasingly being explored as a possible solution for populations with limited access to healthcare. In a systematic review of task shifting for NCD management in low and middle-income countries, Joshi et at ${ }^{45}$ suggest that the use of non-physician health workers may be effective and additionally be cost-effective. There are mixed results for the effectiveness of community health workers $(\mathrm{CHWs})$ in identification and management of cardiovascular disease in various settings in India. ${ }^{46} 47$ Further trials to assess the effectiveness of CHWs in the management of cardiovascular risk factors are currently underway. ${ }^{48}{ }^{49}$ Neither of these trials are testing the exclusive use of ASHAs in specifically delivering hypertension self-management education and monitoring. However, these trials, along with ours, will provide valuable evidence which may encourage the government to fund ASHAs to manage NCDs or to identify a new cadre of village health workers to work in the area of NCDs. Since ASHAs are employed in most rural regions, the programme is more likely to be scalable across rural India. This information will also contribute to disease prevention at a global level as the lessons learnt could be suitably adapted across other similar settings.

\section{Strengths and limitations}

A limitation to this study is that the training programme delivered to ASHAs has not been formally tested. This may limit the efficacy of the intervention. However, after seeking input from ASHAs involved in the pilot training, we were able to make important refinements to the ASHA training booklet and the educational resources for participants. We also assessed the ASHAs skills in measuring anthropometry and $\mathrm{BP}$, as well as their knowledge of hypertension and NCDs before and after the formal training period as well as at the end of the intervention. This will enable us to determine the competencies of the ASHAs.

There are also a number of strengths to this study. First, this is a large community-based study in three economically and developmentally diverse rural populations in Southern India, with detailed data on demographics; lifestyle-related factors, including physical activity, tobacco use and alcohol consumption; dietary factors, including cooking practices and use of salt; stress and overcrowding. Second, there is detailed knowledge about hypertension and its risk factors, awareness of hypertensive status and further details about the use of medications, barriers to treatment, including access, cost, adoption of lifestyle factors and compliance with medication use. Such a rich data set will enable detailed exploration of associations between hypertension and economic and epidemiological transition. Furthermore, the additional qualitative studies, including the medicines availability survey, indepth interviews and focus group discussions, will allow us to triangulate potential barriers to diagnosis, treatment and control of hypertension. Data are collected using validated and standardised tools which will allow comparisons with other studies in India and other LMICs.

Finally, the feasibility study of employing ASHAs in community-based activities to prevent and manage hypertension will add to the increasing body of evidence for using non-physician health workers in the detection and management of NCDs, particularly in LMICs.

\section{Author affiliations}

${ }^{1}$ School of Clinical Sciences at Monash Health, Monash University, Melbourne, Victoria, Australia

${ }^{2}$ The George Institute for Global Health, University of Sydney, Sydney, New South Wales, Australia

${ }^{3}$ Melbourne School of Population and Global Health, University of Melbourne, Melbourne, Victoria, Australia

${ }^{4}$ Department of Cardiology, Westmead Hospital, Sydney, New South Wales, Australia

${ }^{5}$ Achutha Menon Centre for Health Science Studies, Sree Chitra Tirunal Institute for Medical Sciences and Technology, Trivandrum, Kerala, India ${ }^{6}$ School of Public Health and Preventative Medicine, Monash University, Melbourne, Victoria, Australia

${ }^{7}$ Nossal Institute for Global Health, Melbourne School of Population and Global Health, University of Melbourne, Melbourne, Victoria, Australia

${ }^{8}$ Department of Endocrinology, Diabetes and Metabolism, Christian Medical

College, Vellore, Tamil Nadu, India

${ }^{9}$ Department of Physiology, Monash University, Melbourne, Australia

${ }^{10}$ Cardiovascular Disease Program, Biosciences Discovery Institute, Monash University, Melbourne, Australia

${ }^{11}$ Rishi Valley Rural Health Centre, Chittoor District, Andhra Pradesh, India

${ }^{12}$ George Institute for Global Health, Hyderabad, Telangana, India

${ }^{13}$ George Institute for Global Health, Oxford University, Oxford, UK

Contributors MAR wrote the first draft of the manuscript. AGT is the principal investigator, and conceived the study. All authors provided input into the study design, provided intellectual input to the manuscript and approved the final version of the manuscript.

Funding This work was funded by The National Health and Medical Research Council (NHMRC) under the Global Alliance for Chronic Disease (GACD) programme (grant number GNT1040030), with additional funds provided from Monash University (via the principal investigator). AGT reports grants from National Health and Medical Research Council of Australia (GNT1040030 and GNT1042600), and grants from Monash University, during the conduct of the study. PKM reports receiving an Intermediate Career Fellowship from the Wellcome Trust/DBT India Alliance, during the conduct of the study. RJ was supported by a fellowship from the National Heart Foundation (GNT100484). AM, NT and SA report grants from National Health and Medical Research Council of Australia, during the conduct of the study. RGE reports receiving grant funding from the National Health and Medical Research Council of Australia.

Disclaimer The funders had no role in the design or conduct of the study, and no role in the decision to submit the protocol for publication.

Competing interests None declared.

Ethics approval Approval for the overall study was obtained from the Health Ministry's Screening Committee, Ministry of Health \& Family Welfare, 
Government of India (No. 58/4/Indo_CHR/2013/NCD-II) and Monash University Human Research Ethics Committee (MUHREC number 2013001327). Each site obtained ethics approval from individual governing institutional review boards (IRBs) for site specific aspects of the study. The site in Trivandrum obtained approval from Sree Chitra Tirunal Institute for Medical Sciences and Technology Institutional Ethics Committee (IEC registration number ECR/189/Inst/KL/2013). The West Godavari site obtained approval from the Centre for Chronic Disease Control, New Delhi (IEC: IRB00006330). The Rishi Valley site obtained approval from the IRBs of the Rishi Valley Education Centre and Christian Medical Centre, Vellore (IRB Min No. 8313). Prior to inclusion in the survey, Sarpanches are approached to obtain approval for the study team to seek participation from the residents of the village in the study.

Provenance and peer review Not commissioned; externally peer reviewed.

Open Access This is an Open Access article distributed in accordance with the Creative Commons Attribution Non Commercial (CC BY-NC 4.0) license which permits others to distribute, remix, adapt, build upon this work noncommercially, and license their derivative works on different terms, provided the original work is properly cited and the use is non-commercial. See: http:// creativecommons.org/licenses/by-nc/4.0/

\section{REFERENCES}

1. Lim SS, Vos T, Flaxman AD, et al. A comparative risk assessment of burden of disease and injury attributable to 67 risk factors and risk factor clusters in 21 regions, 1990-2010: a systematic analysis for the Global Burden of Disease Study 2010. Lancet 2012;380:2224-60

2. Kearney PM, Whelton M, Reynolds K, et al. Global burden of hypertension: analysis of worldwide data. Lancet 2005;365:217-23.

3. Anchala R, Kannuri NK, Pant $\mathrm{H}$, et al. Hypertension in India: a systematic review and meta-analysis of prevalence, awareness, and control of hypertension. J Hypertens 2014;32:1170-7.

4. Gupta R. Convergence in urban-rural prevalence of hypertension in India. J Hum Hypertens 2016;30:79-82.

5. Thankappan KR, Shah B, Mathur P, et al. Risk factor profile for chronic non-communicable diseases: results of a community-based study in Kerala, India. Indian J Med Res 2010;131:53-63.

6. Thankappan KR, Sivasankaran S, Sarma PS, et al. Prevalence-correlates-awareness-treatment and control of hypertension in Kumarakom, Kerala: baseline results of a community-based intervention program. Indian Heart $J$ 2006;58:28-33.

7. Association of Physicians of India. Indian guidelines on hypertension (I.G.H.)-III. 2013. J Assoc Physicians India 2013;61(2 Suppl): 6-36.

8. Mosley WH, Chen LC. An analytical framework for the study of child survival in developing countries. 1984. Bull World Health Organ 2003;81:140-5

9. Khatib R, Schwalm JD, Yusuf S, et al. Patient and healthcare provider barriers to hypertension awareness, treatment and follow up: a systematic review and meta-analysis of qualitative and quantitative studies. PLOS ONE 2014;9:e84238.

10. Muntner P, Gu D, Wu X, et al. Factors associated with hypertension awareness, treatment, and control in a representative sample of the Chinese population. Hypertension 2004;43:578-85

11. Agyemang C, Bruijnzeels MA, Owusu-Dabo E. Factors associated with hypertension awareness, treatment, and control in Ghana, West Africa. J Hum Hypertens 2005;20:67-71.

12. Ohene Buabeng K, Matowe L, Plange-Rhule J. Unaffordable drug prices: the major cause of non-compliance with hypertension medication in Ghana. J Pharm Pharm Sci 2004;7:350-2.

13. Wang J, Zhang L, Wang F, et al. Prevalence, awareness, treatment and control of hypertension in China: results from a national survey. Am J Hypertens 2014;27:1355-61.

14. Maimaris W, Paty J, Perel P, et al. The influence of health systems on hypertension awareness, treatment, and control: a systematic literature review. PLoS Med 2013;10:e1001490.

15. Powers BJ, Olsen MK, Oddone EZ, et al. Literacy and blood pressure-do healthcare systems influence this relationship? A cross-sectional study. BMC Health Serv Res 2008;8:1-9.

16. Kahn K, Tollman S, Thorogood M, et al. Older adults and the health transition in Agincourt, rural South Africa: new understanding, growing complexity. In: Cohen B, Menken J, eds. Aging in
Sub-Saharan Africa: recommendations for furthering research. Washington, DC: The National Academies Press, 2006:166-88.

17. Prabhakaran D, Chaturvedi V, Shah $\mathrm{P}$, et al. Differences in the prevalence of metabolic syndrome in urban and rural India: a problem of urbanization. Chronic IIIn 2007;3:8-19.

18. Peters DH, Rao KS, Fryatt R. Lumping and splitting: the health policy agenda in India. Health Policy Plan 2003;18:249-60.

19. State Planning Board. Human Development Report: Government of Kerala, 2005. http://planningcommission.nic.in/plans/stateplan/sdr pdf/shdr kerala05.pdf (accessed Apr 2016).

20. Office of the Registrar General of India. Census of India. 2011. http:// www.censusindia.gov.in/2011-prov-results/prov_data_products kerala_.html (accessed Apr 2016)

21. C-14 Five Year Age Group Data By Residence And Sex. Secondary C-14 five year age group data by residence and sex, 2011. http:// www.censusindia.gov.in/2011census/C-series/C-14.html (accessed Apr 2016)

22. Thankappan KR. Some health implications of globalization in Kerala India. Bull World Health Organ 2001;79:892-3.

23. Infochange India. Life Expectancy for Males and Females in Selected States in India. Secondary Life Expectancy for Males and Females in Selected States in India. 2012. http://infochangeindia.org/ public-health/statistics/life-expectancy-for-males-and-females-inselected-states-in-india.html (accessed Apr 2016)

24. Government of India. Census 2011. Secondary Census 2011. 2015. http://www.census2011.co.in/census/district/ (accessed Apr 2016)

25. Thrift AG, Evans RG, Kalyanram K, et al. Gender-specific effects of caste and salt on hypertension in poverty: a population-based study. J Hypertens 2011;29:443-50.

26. World Health Organization. Section 3: Guide to Physical Measurements (Step 2). WHO STEPS Surveillance ManualUpdated: 13 June 2008 2008; (Part 3: Training and Practical Guides). http://www.who.int/chp/steps/Part3_Section3.pdf (accessed Apr 2016)

27. World Health Organization. Global Database on Body Mass Index: BMI classification. Secondary Global Database on Body Mass Index: BMI classification 2016. http://apps.who.int/bmi/index.jsp? introPage=intro_3.html (accessed Jul 2016).

28. Green J, Willis K, Hughes E, et al. Generating best evidence from qualitative research: the role of data analysis. Aust NZ J Public Health 2007;31:545-50.

29. Bartholomew LK, Parcel GS, Kok G, et al. Planning health promotion programs: an intervention mapping approach. 1 edn. San Francisco, CA: Jossey-Bass, 2006.

30. Stokols D. Translating social ecological theory into guidelines for community health promotion. Am J Health Promot 1996;10:282-98.

31. Stokols D, Allen J, Bellingham RL. The social ecology of health promotion: implications for research and practice. Am J Health Promot 1996;10:247-51.

32. World Health Organization. 18th WHO Model List of Essential Medicines, April 2013. http://apps.who.int/iris/bitstream/10665/93142/ 1/EML 18 eng.pdf?ua=1 (accessed Apr 2016)

33. Ministry of Health and Family Welfare. National List of Essential Medicines of India, 2011. http://apps.who.int/medicinedocs/ documents/s18693en/s18693en.pdf (accessed Apr 2016).

34. Health Medical and Family Welfare Department Government of Andhra Pradesh. Essential Medicines List 2010. http://aptribes.gov. in/Health/115_13052010Health.pdf (accessed Apr 2016).

35. World Health Organization and Health Action International. Measuring medicine prices, availability, affordability and price components. 2nd edn. Geneva: World Health Organization, 2008. http://www.who.int/medicines/areas/access/OMS Medicine_prices. pdf (accessed Apr 2016).

36. Lorig KR, Sobel DS, Stewart AL, et al. Evidence suggesting that a chronic disease self-management program can improve health status while reducing hospitalization: a randomized trial. Med Care 1999;37:5-14.

37. Franek J. Self-management support interventions for persons with chronic disease: an evidence-based analysis. Ontario Health Technol Assess Ser 2013;13:1-60. http://www.hqontario.ca/en/ documents/eds/2013/full-report-OCDM-self-management.pdf (accessed Apr 2016)

38. Nyamathi A, Vatsa M, Khakha DC, et al. HIV knowledge improvement among nurses in India using a train-the-trainer program. J Assoc Nurses AIDS Care 2008;19:443-9.

39. Ministry of Health and Family Welfare. Reading Material for ASHA Role in Prevention and Control of Non Communicable Diseases (NCDs) Book No. 8. http://mohfw.nic.in/showfile.php?lid=656 (accessed Apr 2016). 
40. National Rural Health Mission. Community processes guidelines2013. New Delhi, 2013. http://nrhm.gov.in/images/pdf/ communitisation/asha/Orders-Guidelines/Guidelines_for_ Community_Processes 2014_English.pdf (accessed Apr 2016).

41. National Rural Health Mission. Guidelines for village health sanitation and nutrition committee Part B. In: Gupta A, ed. Guidelines for community processes. New Delhi: Ministry of Health and Family Welfare, 2013:29. http://nrhm.gov.in/images/ $\mathrm{pdf} /$ communitisation/vhsnc/order-guidelines/Guidelines_for_ Community_Processes_2014\%20English.pdf (accessed Apr 2016).

42. Kim MT, Hill MN, Bone LR, et al. Development and testing of The Hill-Bone Compliance to High Blood Pressure Therapy Scale. Prog Cardiovasc Nurs 2000;15:90-6.

43. Glasgow RE, Whitesides $\mathrm{H}$, Nelson $\mathrm{CC}$, et al. Use of the patient assessment of chronic illness care (PACIC) with diabetic patients: relationship to patient characteristics, receipt of care, and self-management. Diabetes Care 2005;28:2655-61.

44. McCormack LA, Williams-Piehota PA, Bann CM, et al. Development and validation of an instrument to measure resources and support for chronic illness self-management: a model using diabetes. Diabetes Educ 2008;34:707-18.

45. Joshi R, Alim M, Kengne AP, et al. Task shifting for non-communicable disease management in low and middle income countries-a systematic review. PLOS ONE 2014;9:e103754.

46. Joshi R, Chow CK, Raju PK, et al. The Rural Andhra Pradesh Cardiovascular Prevention Study (RAPCAPS): a cluster randomized trial. J Am Coll Cardiol 2012;59:1188-96.

47. Prabhakaran $D$, Jeemon $\mathrm{P}$, Goenka $\mathrm{S}$, et al. Impact of a worksite intervention program on cardiovascular risk factors: a demonstration project in an Indian industrial population. J Am Coll Cardiol 2009;53:1718-28

48. Fathima FN, Joshi R, Agrawal T, et al. Rationale and design of the Primary pREvention strategies at the community level to Promote Adherence of treatments to pREvent cardiovascular diseases (REPARE) trial. Am Heart J 2013;166:4-12.

49. Jeemon P, Prabhakaran D, Goenka S, et al. Impact of comprehensive cardiovascular risk reduction programme on risk factor clustering associated with elevated blood pressure in an Indian industrial population. Indian J Med Res 2012;135:485-93. 